\title{
Mental Disorders in Nasopharyngeal Carcinoma Patients Receiving Radiation Therapy: A Nationwide Population-based Study
}

\author{
O KYU NOH $\mathrm{NO}^{1,2,3}$ and JAESUNG HEO ${ }^{1}$ \\ ${ }^{1}$ Department of Radiation Oncology, Ajou University School of Medicine, Suwon, Republic of Korea; \\ ${ }^{2}$ Department of Biomedical Informatics, Ajou University School of Medicine, Suwon, Republic of Korea; \\ ${ }^{3}$ Office of Biostatistics, Ajou Research Institute for Innovative Medicine, \\ Ajou University Medical Center, Suwon, Republic of Korea
}

\begin{abstract}
Background/Aim: Using nationwide data from South Korea, we analyzed the prevalence of mental disorders among patients with nasopharyngeal carcinoma (NPC) who received radiotherapy $(R T)$. Patients and Methods: Data were collected between January 2010 and December 2014, and 1,819 patients diagnosed with NPC who underwent RT were identified. We calculated the prevalence of the five most commonly diagnosed mental disorders and their incidences according to a time sequence based on the time of RT. Results: Among 1,819 patients, 144 (7.9\%) were diagnosed at least once with a mental disorder between one year before the start of RT and the last follow-up. Based on the first diagnosis, 51 $(35.4 \%)$ patients experienced anxiety, and 46 (31.9\%) suffered from depression. The frequency of mental disorders increased up to the beginning of RT and then declined. Before the start of $R T$, anxiety was most frequent, and depression occurred after the start of RT. The cumulative incidence of mental disorders in the older age group tended to be higher than that of the younger group ( $\geq 54$ vs. $<54$ years, log-rank $p=0.052)$. Conclusion: The proportion of mental disorders differed before and after the start of RT in patients with NPC. At the beginning of $R T$, mental disorders emerged most often during management. Early screenings and interventions for mental disorders were able to improve quality of life (QOL).
\end{abstract}

This article is freely accessible online.

Correspondence to: Jaesung Heo, MD, Ph.D., Department of Radiation Oncology, Ajou University School of Medicine, 164 Worldcup-ro, Yeongtong-gu, Suwon 16499, Republic of Korea. Tel: +82 312195885, Fax: +82 312195894, e-mail: md.js.informatics@gmail.com

Key Words: Nasopharyngeal carcinoma, mental disorder, radiation therapy, quality of life, claim data.
Nasopharyngeal carcinoma (NPC) is rare $(1,2)$. However, its incidence shows geographical variations (3). While the incidence rate is low (0.5-2 cases/100,000 person-year) in Western countries (4), it has reached 3.09 cases/100,000 person-year in high-risk areas, such as China (5).

During treatment for NPC, patients may experience treatment-related complications, that can deteriorate their quality of life (QOL). Particularly, radiation therapy (RT) with/without chemotherapy, a standard of care with curative intent for NPC (6), can lead to psychiatric comorbidities as well as physical complications (7). Due to site specificity, patients with NPC (head and neck cancer) are more susceptible to emotional trauma than those who have malignancies at other sites (8). Moreover, physical changes during treatment can aggravate the psychosocial status of NPC survivors (9).

A low QOL is related to psychosocial factors that cause anxiety, depression, and fear of recurrence among head and neck cancer survivors $(10,11)$. Psychological problems can also decrease compliance and result in increased mortality (12). Therefore, it is essential to understand the risks and patterns of psychiatric comorbidities in the treatment of patients with NPC. Moreover, adequate clinical support for mental problems could enhance prognosis (13).

A previous study investigated mental disorders among patients with NPC; however, it was limited to a small sample size and had a cross-sectional design (14). It examined the prevalence of mental disorders and its longitudinal pattern according to a time sequence (before, during, and after RT) in patients with NPC using national claim data from South Korea.

\section{Patients and Methods}

We generated the NPC cohort based on claim data from the Health Insurance Review and Assessment Service (HIRA), gathered between January 2010 and December 2014. The HIRA claim data are from the public medical insurance system, which covers $90 \%$ of South Korea's 
in vivo $35: 2901-2908(2021)$

Table I. Frequency of mental disorders in patients with nasopharyngeal carcinoma $(N=1,819)$.

\begin{tabular}{|c|c|c|c|c|c|c|c|}
\hline \multirow[t]{2}{*}{ Age (year) } & \multicolumn{6}{|c|}{ No. of patients (\%) } & \multirow[b]{2}{*}{$\begin{array}{c}\text { Substance } \\
\text { abuse }\end{array}$} \\
\hline & $\begin{array}{l}\text { Nasopharyngeal } \\
\text { carcinoma }\end{array}$ & $\begin{array}{l}\text { Mental } \\
\text { disorder }\end{array}$ & $\begin{array}{l}\text { Depressive } \\
\text { disorder }\end{array}$ & $\begin{array}{l}\text { Anxiety } \\
\text { disorder }\end{array}$ & $\begin{array}{l}\text { Stress/adjustment } \\
\text { disorder }\end{array}$ & $\begin{array}{c}\text { Somatoform/conversion } \\
\text { disorder }\end{array}$ & \\
\hline $10-39$ & 257 & $18(7.0)$ & $7(2.7)$ & $6(2.3)$ & $2(0.8)$ & $2(0.8)$ & $1(0.4)$ \\
\hline $40-49$ & 386 & $27(7.0)$ & $5(1.3)$ & $13(3.4)$ & $1(0.3)$ & $6(1.6)$ & $2(0.5)$ \\
\hline $50-59$ & 570 & $41(7.2)$ & $14(2.5)$ & $13(2.3)$ & $4(0.7)$ & $8(1.4)$ & $2(0.4)$ \\
\hline $60-69$ & 384 & $35(9.1)$ & $10(2.6)$ & $12(3.1)$ & $6(1.6)$ & $4(1.0)$ & $3(0.8)$ \\
\hline $70-99$ & 222 & $23(10.4)$ & $10(4.5)$ & $7(3.2)$ & $1(0.5)$ & $5(2.3)$ & $0(0.0)$ \\
\hline Total & 1,819 & $144(7.9)$ & $46(2.5)$ & $51(2.8)$ & $14(0.8)$ & $25(1.4)$ & $8(0.4)$ \\
\hline
\end{tabular}

population. The HIRA data consist of reimbursement claims for healthcare services received during outpatient visits and inpatient admissions to medical institutions. We were able to extract general information, the healthcare services provided, the diagnosis, the outpatient prescription, drug information, and provider information.

We used the diagnostic code of NPC (C11) according to the sixth edition of the International Classification of Disease (ICD-10). We verified the use of RT based on the codes from the HIRA claim data (HD061, 3-dimensional conformal therapy; HZ271, intensity modulated radiation therapy). We identified the following five mental disorders using the ICD-10 codes: 1) depression (F32, F33); 2) anxiety (F40, F41); 3) stress/adjustment disorder (F43); 4) somatoform/conversion disorder (F44, F45); and 5) substance abuse disorder (F10-F19); these are the most commonly diagnosed mental illnesses among cancer patients $(15,16)$. To evaluate the type of treatment, we used the corresponding codes for medication (antipsychotic drugs, antidepressants, mood stabilizers, antianxiety drugs) and psychotherapy (individual psychotherapy: NN011-NN013, group psychotherapy: NN021-NN023, family therapy: NN031-NN032). Regarding the intensity of psychotherapy and medication, we counted the number of prescriptions for psychotherapy and medication.

We estimated the prevalence of mental disorders and its longitudinal pattern according to a time sequence (before, during, and after RT). We limited our observations to within one year before $\mathrm{RT}$ in order to focus on the changes in mental disorders around the time of RT. We employed descriptive statistics to assess the frequency of mental disorders. We examined the characteristics of mental disorders according to age, sex, and the use of chemotherapy. We calculated the cumulative incidence of psychiatric disorders using the Kaplan-Meier method, and we compared the curves between the variables through the log-rank test. All statistical analyses were performed with R 3.0.2.

We received approval from the institutional review board (IRB) of Ajou University Hospital (IRB No. AJIRB-MED-EXP-16-494) and retrospectively analyzed de-identified claim data. The data used in the study are potentially identifiable and are not publicly available. The raw claim datasets generated and/or analyzed during the current study are not publicly available due to ethical restrictions by the authorizing HIRA.

\section{Results}

We identified a total of 1,819 patients with NPC who received RT between January 2010 and December 2014 in South Korea.
Of these, $144(7.9 \%)$ were diagnosed at least once with a mental illness between one year before the start of RT and at the time of last follow-up. The median age of all patients was 54 years, and for those with mental disorders, it was 56 years. Table I summarizes the frequencies of mental disorders. Of the 144 abovementioned patients, 51 (35.4\%) and $46(31.9 \%)$ experienced anxiety and depression, respectively. Twenty-five (17.4\%) experienced somatoform/conversion disorder.

Figure 1 illustrates the frequencies of mental disorders between the periods before and after RT. Before and after the start of RT, 57 and 87 patients were diagnosed with mental disorders as their first diagnosis. During the pre-RT period, anxiety was the most common disorder $(40.4 \%)$, followed by somatoform/conversion disorder $(29.8 \%)$. After the start of RT, depression was the ailment most often identified. The proportion of patients with depression after the start of RT rose to $43.7 \%$, compared to $14.0 \%$ in the pre-RT period. The ratios of anxiety, substance abuse, and somatoform/conversion disorders fell after the start of RT compared to before RT.

The overall frequency of mental disorders increased until the start of RT and peaked, then declined just after the start of RT (Figure 2). The timing of the peak frequency of each mental disorder was different. The peaks of somatoform/conversion and substance abuse were before the start of RT, while depression and stress/adjustment disorder peaked after the launch of RT. Anxiety reached its peak just at the beginning of RT.

The patterns of mental ailments differed among the age groups (Figure 3). Those in their forties showed abrupt changes around the beginning of RT. A stable incidence of mental disorders was observed in the group of patients who were over 70 . The proportion of anxiety was relatively high among those in their forties compared to the other age group. In the group of those over 70, the proportion of depression was relatively high (Figure 4).

The cumulative incidence of mental disorders in the older group tended to be higher than that of the younger group ( $\geq 54$ vs. $<54$ years, log-rank $p=0.052$ ) (Figure 5A). The cumulative incidence did not vary according to $\operatorname{sex}(p=0.117)$ (Figure $5 \mathrm{~B}$ ) or use of chemotherapy ( $p=0.360)$ (Figure 5C). 


\section{A}

40

35

30

25

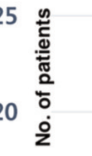

15

10

0

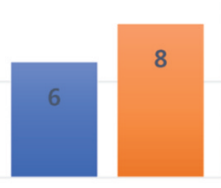

Before RT

- Substance abuse

Anxiety disorder

- Somatoform /conversion disorder

B

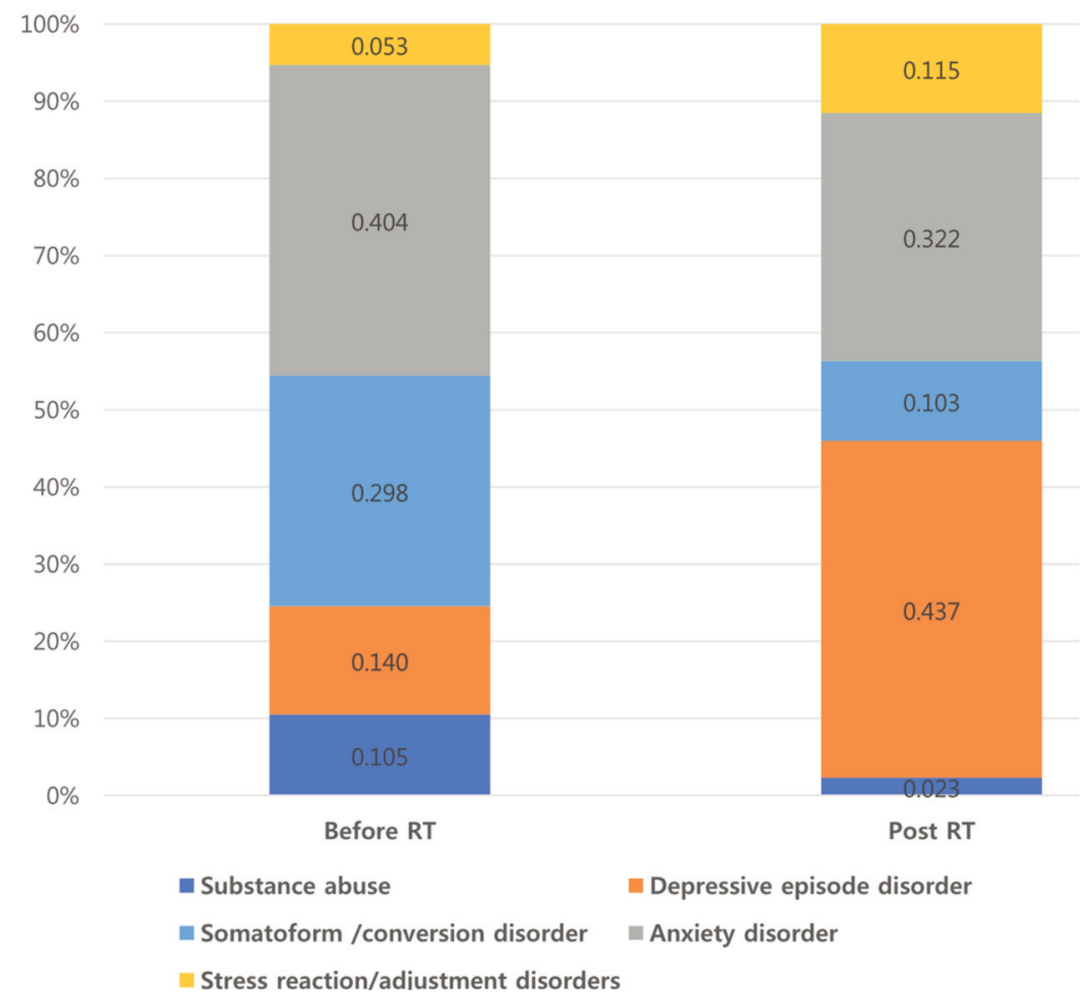

Figure 1. Mental disorders before and after radiation therapy in patients with nasopharyngeal carcinoma. (A) Number of patients with each mental disorder. (B) Proportion of patients with each mental disorder. 


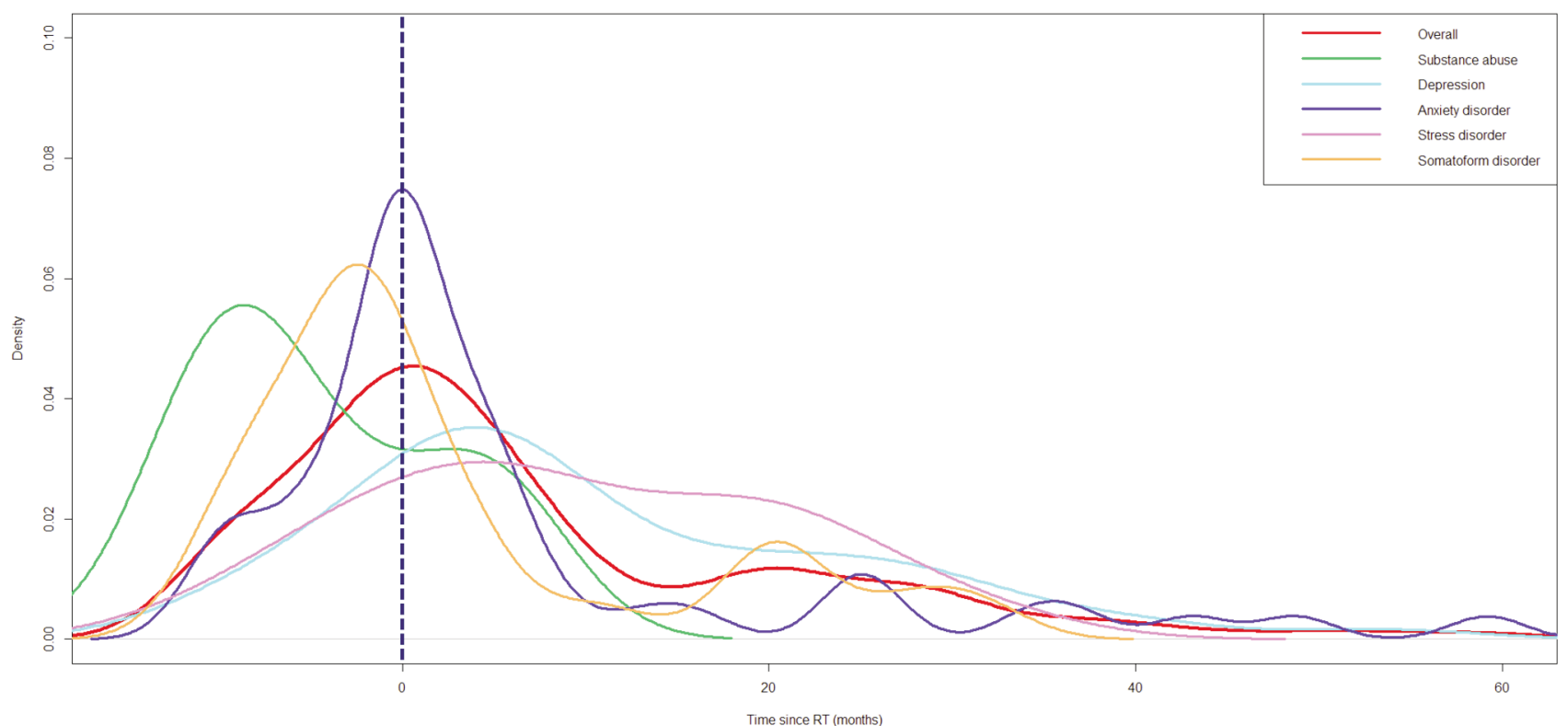

Figure 2. The frequency density plots of mental disorders in patients with nasopharyngeal carcinoma.

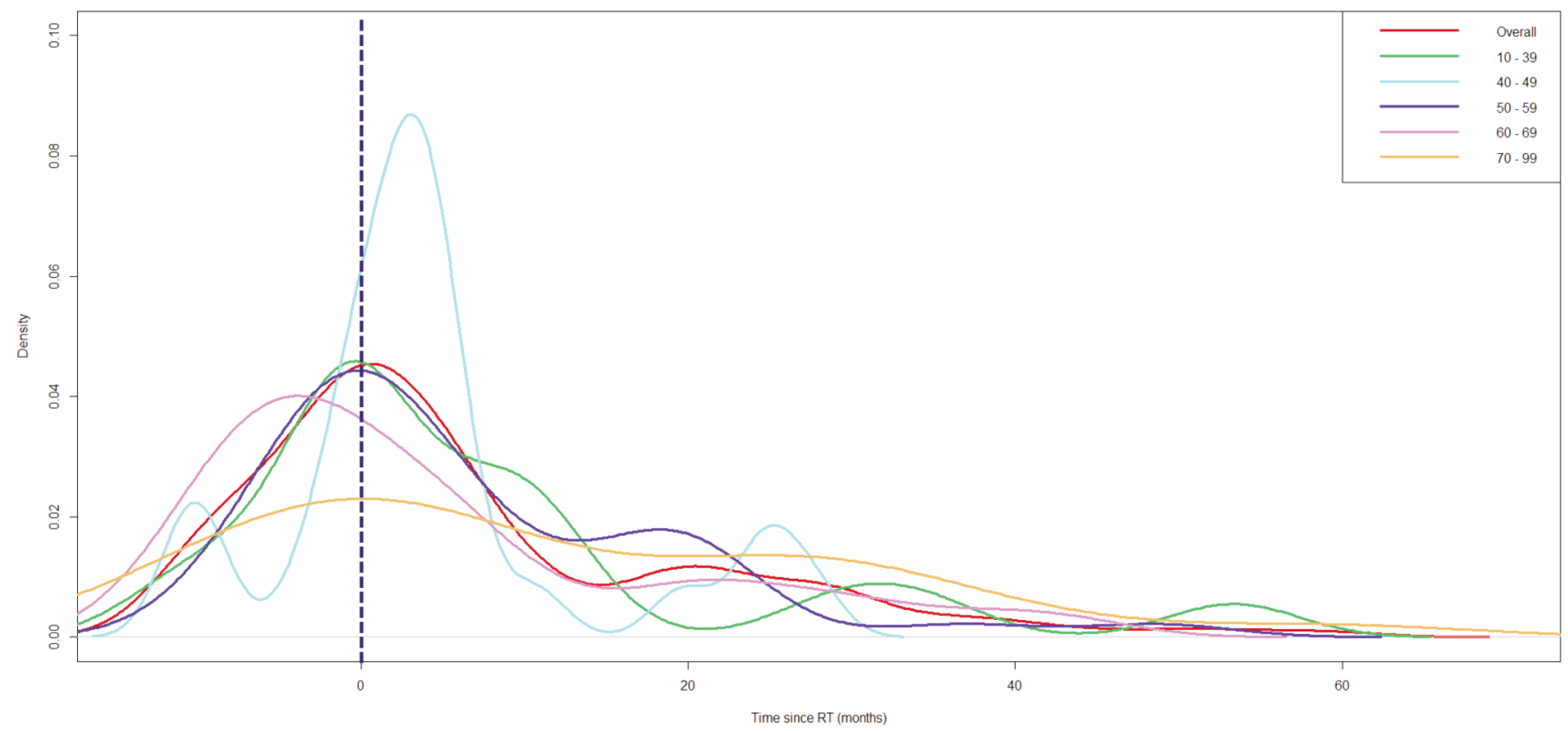

Figure 3. The frequency density plots of mental disorders by age in patients with nasopharyngeal carcinoma.

Table II lists the treatments for mental disorders. Of the 144 patients, $46(31.9 \%)$ were prescribed psychotherapy with medication. Thirty-seven $(25.7 \%)$ received psychotherapy only, and $20(13.9 \%)$ were treated with medication alone. Forty-one $(28.5 \%)$ received no treatment. The median numbers of psychotherapy and medication claims for each patient were 2 (range, 1-106), respectively.
Table III displays the prescription frequency of each treatment for each patient.

\section{Discussion}

Using national data, we found the prevalence of five mental disorders to be $7.9 \%$ in patients with NPC who received RT. 


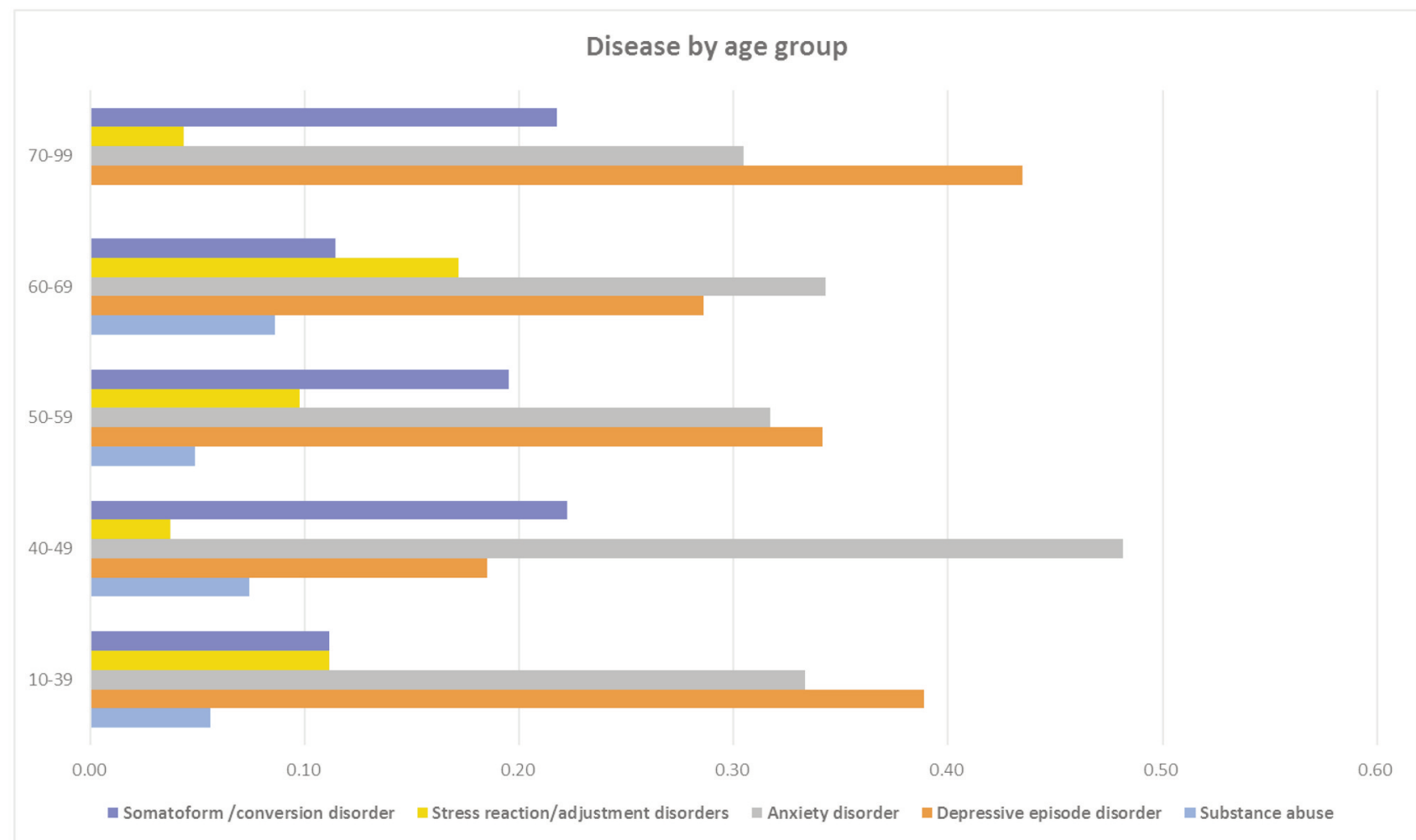

Figure 4. The distribution of mental disorders by age group in patients with nasopharyngeal carcinoma.

Although the claim data tended to underestimate the incidence, our results showed that certain patients with NPC who received RT experienced mental disorders during their illness. Anxiety was the most common (35.4\%) of mental disorders, followed by depression (31.9\%). Anxiety and depression were associated with the QOL of cancer survivors $(17,18)$, including head and neck cancer $(10)$.

The incidences of anxiety and depression differed according to the time sequence. Anxiety was most common before RT $(40.4 \%)$, and fell after the start of RT (32.2\%) (Figure 1B). The most frequent time of anxiety was at the beginning of RT (Figure 2). Patients tended to be apprehensive about their diagnosis, treatment, and prognosis. Before RT, the simulation can increase anxiety with immobilization devices, while the wait time for RT can escalate the level of anxiety. Anxiety is aggravated when patients are left alone in the treatment room during RT. Even after RT is complete, the risk of anxiety may persist owing to the fear of recurrence, decreased performance, and long-term complications. Anxiety could also decrease the patient's immune status (19) and induce non-adherence to treatment (12), which could possibly negate the treatment outcome. Therefore, it is necessary to identify and manage the risk of anxiety at every phase of management in order to improve clinical outcomes as well as QOL. During the simulation for RT, music therapy can help lower anxiety in patients with NPC (20), The relief of distress during the simulation can improve the accuracy of RT while minimizing the set-up error. The proper management of anxiety also boosts treatment compliance.

Unlike anxiety, the proportion of depression was higher after the start of RT (43.7\%) than before RT (14.0\%) (Figure 1). Depression rose over time; its incidence peaked at one month after the start of RT and then declined (Figure 2). The peak incidence for depression at one month seemed to be associated with RT-related acute complications. Dry mouth, pain, and swallowing difficulties due to mucositis, as well as loss of taste, deteriorate QOL and contribute to the occurrence of depression. Hence, proper management of such side-effects and psychosocial support are helpful in mitigating the level of psychiatric distress, including depression. The risk of suicide can be problematic in depression. The rate of suicide is three times higher in head and neck cancer patients than in the general population (21). Thus, the risk of suicide should be screened in NPC patients with depression.

The patterns of mental disorders varied among the age subgroups. In all patients, the frequency of mental disorders increased before RT and reached its peak around the start of RT, then fell afterward. However, patients between 40 and 

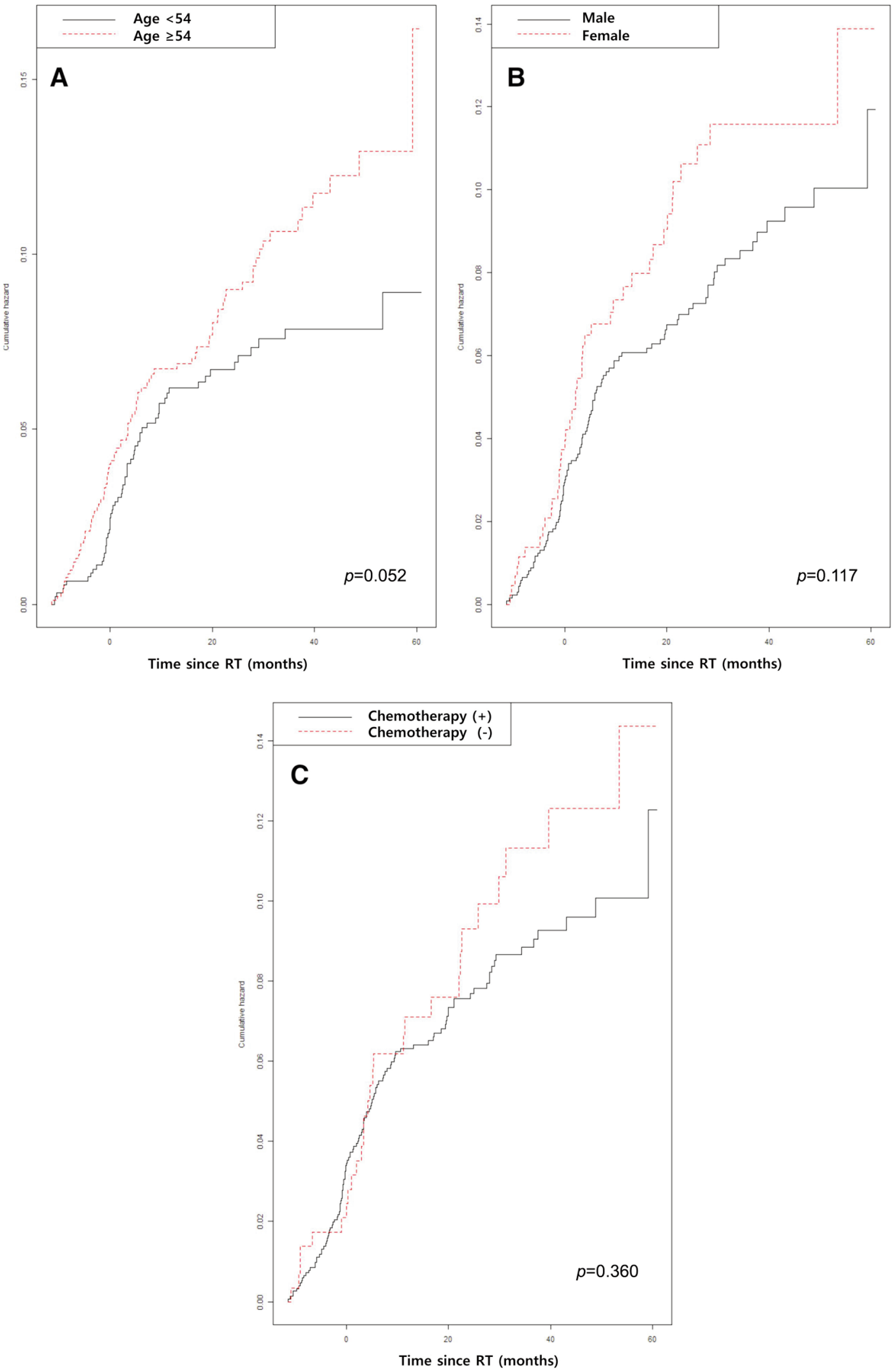

Figure 5. The cumulative incidence of mental disorders by; (A) age, (B) sex, (C) the use of chemotherapy in patients with nasopharyngeal carcinoma. 
Table II. Treatment for mental disorders in patients with nasopharyngeal carcinoma $(N=144)$.

\begin{tabular}{lc}
\hline Type of treatment & No. of patients $(\%)$ \\
\hline Psychotherapy only & $37(25.7)$ \\
Medication only & $20(13.9)$ \\
Psychotherapy+medication & $46(31.9)$ \\
No treatment & $41(28.5)$ \\
\hline
\end{tabular}

Table III. Prescription frequency of treatments for mental disorders in patients with nasopharyngeal carcinoma.

\begin{tabular}{lcc}
\hline \multirow{2}{*}{ No. of prescription } & \multicolumn{2}{c}{ No. of patients (\%) } \\
\cline { 2 - 3 } & Psychotherapy $(\mathrm{N}=83)$ & Medication (N=66) \\
\hline 1 & $39(47.0)$ & $35(53.0)$ \\
$2-5$ & $23(27.7)$ & $20(30.3)$ \\
$6-9$ & $9(10.8)$ & $3(4.5)$ \\
10 & $12(14.5)$ & $8(12.1)$ \\
\hline
\end{tabular}

49 showed an abrupt spike in mental disorders just after beginning RT; the peak occurred two months after the start of RT (Figure 3). Moreover, the proportion of anxiety disorder in this group was relatively higher than that of the other age groups (Figure 4). These results are consistent with the finding that anxiety peaks in middle-aged people (22). Middle-aged women may be more susceptible to mental ailments due to hormonal changes. Moreover, patients of this actively working age group may experience mental stress upon returning to work. Thus, more attention should be taken in terms of handling mental disorders in this age group.

We found that the incidence of mental disorders in the older age group tended to be higher than that of the younger group ( $p=0.052$ ) (Figure 5A). Due to medical comorbidities, elderly patients have a poor prognosis (23). Physical difficulties could make them more susceptible to psychiatric distress than younger patients. A higher incidence of mental disorders in elderly patients also occurs for other types of cancer (24). In our study, although female patients appeared to be more susceptible, there was no significant difference compared to male patients $(p=0.117)$ (Figure $5 \mathrm{~B})$. The use of chemotherapy can affect the incidence of mental disorders because of its added treatment intensity with RT. However, this was not a significant factor in the incidence of mental disorders $(p=0.360)$ (Figure 5C). Although we were not able to pinpoint the vital factors affecting mental disorders in patients with NPC, future studies should investigate them.

Among the 144 patients diagnosed with mental disorders, $41(28.5 \%)$ did not receive psychotherapy or medication (Table II). This implies that many patients were not adequately treated after being diagnosed with mental disorders. The stigma surrounding mental illness in South Korea could be one reason for the relatively high rate of those untreated (25). Concerned about possibly being ostracized, many patients often refuse to receive therapy. Even for those treated with medication, nearly $50 \%$ were prescribed therapy only once (Table III). This low frequency suggests that they may be reluctant to receive ongoing management. Compared to those who took medication, those who received psychotherapy seemed to be treated more consistently. This treatment pattern of mental disorders hints that there is a barrier to the use of mental health services, such as the stigma around mental disorders. Further efforts should be taken to lower this barrier and promote mental health in cancer patients.

Our study had certain limitations. The claim data only served codes for reimbursements based on demographic information such as age, sex, and local area. With such limited clinical data, we could not perform our analysis considering clinical factors such as staging, histology, and the severity of the mental illness. Moreover, compared to interview-based studies, we may have underestimated the prevalence in our study due to the cultural reluctance to be treated for mental disorders in South Korea. The ICD-10 codes do not guarantee that psychiatrists diagnosed the mental disorders identified in this study. However, in most cases, the diagnoses are carefully coded by psychiatric specialists owing to the stigma surrounding mental illness. Moreover, strict surveillance of health insurance companies is mandatory because of the additional financial support provided by the government to treat mental illness. Hence, the codes for mental disorders are relatively reliable in South Korean claim data.

In conclusion, the incidence of mental disorders peaked at the beginning of RT, and the proportion of each mental disorder differed before and after the start of RT. Since mental disorders may be associated with poor QOL, early screening and customized interventions could improve QOL in patients suffering from NPC who are treated with RT.

\section{Conflicts of Interest}

The Authors declare that they have no conflicts of interest.

\section{Authors' Contributions}

Study concept and design: JH, OKN. Acquisition of data: JH. Data analysis: JH. Results interpretation and drafting of the manuscript: JH. Critical revision of the manuscript for important intellectual content: OKN.

\section{Acknowledgements}

This research was supported by the Bio \& Medical Technology Development Program of the National Research Foundation (NRF) funded by the Korean government (MSIT) (No. 2020M3A9E8024907). 


\section{References}

1 Chang ET and Adami HO: The enigmatic epidemiology of nasopharyngeal carcinoma. Cancer Epidemiol Biomarkers Prev 15(10): 1765-1777, 2006. PMID: 17035381. DOI: 10.1158/ 1055-9965.EPI-06-0353

2 Torre LA, Bray F, Siegel RL, Ferlay J, Lortet-Tieulent J and Jemal A: Global cancer statistics, 2012. CA Cancer J Clin 65(2): 87-108, 2015. PMID: 25651787. DOI: 10.3322/caac. 21262

3 Chua MLK, Wee JTS, Hui EP and Chan ATC: Nasopharyngeal carcinoma. Lancet 387(10022): 1012-1024, 2016. PMID: 26321262. DOI: 10.1016/S0140-6736(15)00055-0

4 Wang Y, Zhang $\mathrm{Y}$ and Ma S: Racial differences in nasopharyngeal carcinoma in the United States. Cancer Epidemiol 37(6): 793-802, 2013. PMID: 24035238. DOI: 10.1016/j.canep.2013.08.008

5 Wei KR, Zheng RS, Zhang SW, Liang ZH, Li ZM and Chen WQ: Nasopharyngeal carcinoma incidence and mortality in China, 2013. Chin J Cancer 36(1): 90, 2017. PMID: 29122009. DOI: $10.1186 / \mathrm{s} 40880-017-0257-9$

6 Chen QY, Wen YF, Guo L, Liu H, Huang PY, Mo HY, Li NW, Xiang YQ, Luo DH, Qiu F, Sun R, Deng MQ, Chen MY, Hua YJ, Guo X, Cao KJ, Hong MH, Qian $\mathrm{CN}$ and Mai HQ: Concurrent chemoradiotherapy vs radiotherapy alone in stage II nasopharyngeal carcinoma: phase III randomized trial. J Natl Cancer Inst 103(23): 1761-1770, 2011. PMID: 22056739. DOI: 10.1093/jnci/djr432

7 Djan R and Penington A: A systematic review of questionnaires to measure the impact of appearance on quality of life for head and neck cancer patients. J Plast Reconstr Aesthet Surg 66(5): 647-659, 2013. PMID: 23394687. DOI: 10.1016/j.bjps.2013.01.007

8 Björklund M, Sarvimäki A and Berg A: Living with head and neck cancer: a profile of captivity. Journal of Nursing and Healthcare of Chronic Illness 2(1): 22-31, 2021. DOI: 10.1111/j.1752-9824.2010.01042.x

9 So WK, Chan RJ, Chan DN, Hughes BG, Chair SY, Choi KC and Chan CW: Quality-of-life among head and neck cancer survivors at one year after treatment - a systematic review. Eur J Cancer 48(15): 2391-2408, 2012. PMID: 22579456. DOI: 10.1016/j.ejca.2012.04.005

10 Kohda R, Otsubo T, Kuwakado Y, Tanaka K, Kitahara T, Yoshimura $\mathrm{K}$ and Mimura M: Prospective studies on mental status and quality of life in patients with head and neck cancer treated by radiation. Psychooncology 14(4): 331-336, 2005. PMID: 15655808. DOI: 10.1002/pon.892

11 Hassanein KA, Musgrove BT and Bradbury E: Psychological outcome of patients following treatment of oral cancer and its relation with functional status and coping mechanisms. J Craniomaxillofac Surg 33(6): 404-409, 2005. PMID: 16253509. DOI: $10.1016 /$ j.jcms.2005.05.005

12 Theofilou P and Panagiotaki H: A literature review to investigate the link between psychosocial characteristics and treatment adherence in cancer patients. Oncol Rev 6(1): e5, 2012. PMID: 25992207. DOI: 10.4081/oncol.2012.e5

13 Luckett T, Britton B, Clover K and Rankin NM: Evidence for interventions to improve psychological outcomes in people with head and neck cancer: a systematic review of the literature. Support Care Cancer 19(7): 871-881, 2011. PMID: 21369722. DOI: $10.1007 / \mathrm{s} 00520-011-1119-7$
14 Chiou WY, Lee MS, Ho HC, Hung SK, Lin HY, Su YC and Lee CC: Prognosticators and the relationship of depression and quality of life in head and neck cancer. Indian J Cancer 50(1): 14-20, 2013. PMID: 23713039. DOI: 10.4103/0019-509X.112279

15 Mehnert A, Brähler E, Faller H, Härter M, Keller M, Schulz H, Wegscheider K, Weis J, Boehncke A, Hund B, Reuter K, Richard M, Sehner S, Sommerfeldt S, Szalai C, Wittchen HU and Koch U: Four-week prevalence of mental disorders in patients with cancer across major tumor entities. J Clin Oncol 32(31): 35403546, 2014. PMID: 25287821. DOI: 10.1200/JCO.2014.56.0086 $16 \mathrm{Lu} \mathrm{D}$, Andersson TM, Fall K, Hultman CM, Czene K, Valdimarsdóttir $\mathrm{U}$ and Fang F: Clinical diagnosis of mental disorders immediately before and after cancer diagnosis: A nationwide matched cohort study in Sweden. JAMA Oncol 2(9): 1188-1196, 2016. PMID: 27124325. DOI: 10.1001/jamaoncol. 2016.0483

17 Hutter N, Vogel B, Alexander T, Baumeister H, Helmes A and Bengel J: Are depression and anxiety determinants or indicators of quality of life in breast cancer patients? Psychol Health Med 18(4): 412-419, 2013. PMID: 23116204. DOI: 10.1080/1354 8506.2012.736624

18 Graça Pereira M, Figueiredo AP and Fincham FD: Anxiety, depression, traumatic stress and quality of life in colorectal cancer after different treatments: A study with Portuguese patients and their partners. Eur J Oncol Nurs 16(3): 227-232, 2012. PMID: 21783416. DOI: 10.1016/j.ejon.2011.06.006

19 Miller AH: Neuroendocrine and immune system interactions in stress and depression. Psychiatr Clin North Am 21(2): 443-463, 1998. PMID: 9670236. DOI: 10.1016/s0193-953x(05)70015-0

20 Rossetti A, Chadha M, Torres BN, Lee JK, Hylton D, Loewy JV and Harrison LB: The impact of music therapy on anxiety in cancer patients undergoing simulation for radiation therapy. Int J Radiat Oncol Biol Phys 99(1): 103-110, 2017. PMID: 28816136. DOI: 10.1016/j.ijrobp.2017.05.003

21 Kam D, Salib A, Gorgy G, Patel TD, Carniol ET, Eloy JA, Baredes S and Park RC: Incidence of suicide in patients with head and neck cancer. JAMA Otolaryngol Head Neck Surg 141(12): 1075-1081, 2015. PMID: 26562764. DOI: 10.1001/ jamaoto.2015.2480

22 Bansal P, Chaudhary A, Soni RK, Sharma S, Gupta VK and Kaushal P: Depression and anxiety among middle-aged women: A community-based study. J Family Med Prim Care 4(4): 576581, 2015. PMID: 26985419. DOI: 10.4103/2249-4863.174297

23 Leu Y, Chang Y, Lee J, Lo A, Chen Y and Chen H: Prognosis of nasopharyngeal carcinoma in the elderly is worse than in younger individuals-Experience of a Medical Institute. International Journal of Gerontology 8(2): 81-84, 2019. DOI: 10.1016/j.ijge.2013.08.008

24 Heo J, Chun M, Oh YT, Noh OK and Kim L: Psychiatric comorbidities among ovarian cancer survivors in South Korea: A nationwide population-based, longitudinal study. Psychooncology 27(3): 1021-1026, 2018. PMID: 29280223. DOI: 10.1002/pon.4628

25 Park $J$ and Jeon M: The stigma of mental illness in Korea. Journal of Korean Neuropsychiatric Association 55(4): 299, 2020. DOI: $10.4306 /$ jknpa.2016.55.4.299

Received May 31, 2021

Revised June 23, 2021

Accepted July 14, 2021 